\title{
Experimental verification of selection rules for circularly polarized high harmonics from a solid
}

\author{
Nariyuki Saito*, Peiyu Xia, Faming Lu, Nobuhisa Ishii, Teruto Kanai, and Jiro Itatani \\ The institute for Solid State Physics, the University of Tokyo, 5-1-5 Kashiwanoha, Kashiwa 277- \\ 8581, Japan
}

\begin{abstract}
We experimentally verify selection rules for circularly polarized high harmonics from solids by using single-color circularly polarized midinfrared pulses. Our result offers a novel way to produce circularly polarized, coherent short-wavelength light.
\end{abstract}

\section{Introduction}

Recently, circularly polarized high harmonics (HHs) have attracted great attention because of their potential applicability to ultrafast spectroscopy of magnetic materials and chiral molecules. Until now, circularly polarized high-harmonic generation (HHG) from gas atoms has been realized by using counter-rotating two-color [1] or counter-rotating noncollinearly overlapped beams [2]. Meanwhile, it has been theoretically predicted that circularly polarized HHs can be generated by irradiating single-color circularly polarized optical pulses to an HHG medium with rotational symmetry, and that their spectrum and the polarization obey simple selection rules [3, 4]. For an HHG medium with $N$-fold rotational symmetry, the selection rules are given by

$$
n=\sigma+N j(j \in \mathbb{N}) \text {. }
$$

Here, $n$ is the harmonic order, $\sigma= \pm 1$ represents the state of circular polarization of the $n$ th harmonic ( $\sigma=1$ means circular polarization with the same helicity as the fundamental pulse,

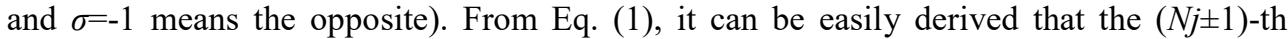
harmonics are allowed with counter-rotating circular polarization and that the other harmonics are forbidden (Table 1). The selection rules have been experimentally verified for perturbative SHG or THG [5, 6], but not for non-perturbative HHG. This is because, in the case of HHG in gaseous media, circularly polarized laser pulses cannot generate HHs. According to the three-step mechanism of gas HHG, a tunnel-ionized electron recollides with its parent ion to produce a $\mathrm{HH}$ photon. If a single-color circular field is induced to a gas atom, the ionized electron does not return to its parent ion. An alternative approach exists in the case of solid HHG. The mechanism of solid HHG includes intraband current or electron collision with adjacent atoms [7], where a tunnel-ionized electron does not return to its parent ion. In fact, You et al. observed $\mathrm{HHG}$ from crystalline $\mathrm{MgO}$ using single-color circularly polarized pulses [7].

\footnotetext{
${ }^{*}$ Corresponding author: nariyuki.saito@issp.u-tokyo.ac.jp
} 
Here, we report on the experimental verification of the selection rules. We observe up to the eighth harmonic from a GaSe crystal with a single-color, circularly polarized mid-infrared (MIR) drive laser. The polarization states of the HHs are analyzed and shown to be consistent with the selection rules [8].

Table 1. Selection rules for circularly polarized HHG driven by right-circularly polarized pulses.

\begin{tabular}{cccccccccc}
\hline \hline & \multicolumn{8}{c}{ Harmonic order } \\
\cline { 2 - 9 } $\begin{array}{c}\text { Rotational } \\
\text { symmetry }\end{array}$ & 2 & 3 & 4 & 5 & 6 & 7 & 8 & 9 \\
\hline 2-fold & - & E & - & E & - & E & - & E \\
3-fold & L & - & R & L & - & R & L & - \\
4-fold & - & L & - & R & - & L & - & R \\
6-fold & - & - & - & L & - & R & - & - \\
\hline \hline
\end{tabular}

*E: elliptic, L: left-circularly polarized, R: right-circularly polarized, -: forbidden.

\section{Experimental setup}

Figure 1(a) illustrates a schematic of the experimental setup. We use MIR pulses from a home-built KTA-based optical parametric amplifier $(3.5 \mu \mathrm{m}, 50 \mathrm{~J}, 70 \mathrm{fs}, 300 \mathrm{~Hz})$. The MIR pulse energy and polarization are adjusted by two wire grid polarizers and a quarter-wave plate, and focused into a 30- m-thick GaSe crystal with threefold rotational symmetry $(N=3$ in Eq. (1)). Figure 1(b) shows the crystal structure of GaSe and MIR propagation direction. The emitted HHs are recorded with an optical multichannel analyzer. A quarter-wave Fresnel rhomb retarder and a wire grid polarizer are optionally inserted after the GaSe crystal to resolve the polarization of the HHs.

(a)

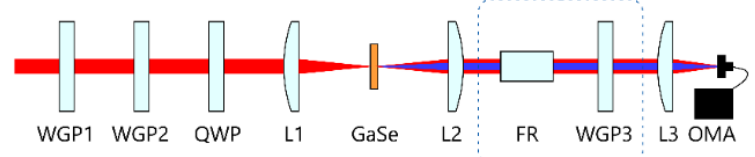

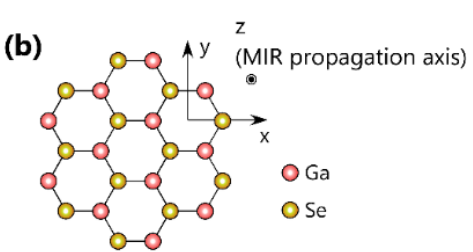

Fig. 1. (a) Schematic of the experimental setup (WGP, wire grid polarizer; QWP, quarter-wave plate; L, lens; FR, quarter-wave Fresnel rhomb retarder; OMA, optical multichannel analyzer). Optics in dotted lines are used for polarization analysis. (b) Crystal structure of GaSe. The MIR beam is propagated along the $\mathrm{z}$-axis.

\section{Results and discussion}

Figure 2(a) represents the total HH spectra under linear (blue curve) and circular (red curve) excitation. Here, the MIR pulse energy is adjusted to $23.0 \mathrm{~J}$, corresponding to peak electric field of $11.2 \mathrm{MV} / \mathrm{cm}$ and $7.9 \mathrm{MV} / \mathrm{cm}$ for linear and circular polarization, respectively. The third, sixth, (and possibly ninth) harmonics disappear in the case of circular excitation, while the other harmonics maintain similar yields. Figure 2(b) represents the left-circularly polarized (LCP, blue curve) and right-circularly-polarized (RCP, red curve) components of the HHs under RCP excitation with the same pulse energy. The second, fifth, and eighth harmonics are predominantly LCP, while the fourth and seventh harmonics are RCP. We confirm that the ellipticities of these HHs are more than 0.9. All these observations are consistent with the theoretically predicted selection rules. In addition, in order to confirm that our experimental condition is in the non-perturbative regime, we measure the intensity 
dependence of thse $\mathrm{HH}$ yield with circular excitation. A clear deviation from the perturbative scaling, where the $n$-th $\mathrm{HH}$ yield scales as the $2 n$-th power of the peak electric field, is observed below $7 \mathrm{MV} / \mathrm{cm}$.

In conclusion, we experimentally verify selection rules for circularly polarized HHs from a crystalline solid irradiated by a single-color circularly polarized MIR pulses. This work opens the doorway to produce and control circularly polarized HHs that can be extended to the extreme ultraviolet and the attosecond regime.
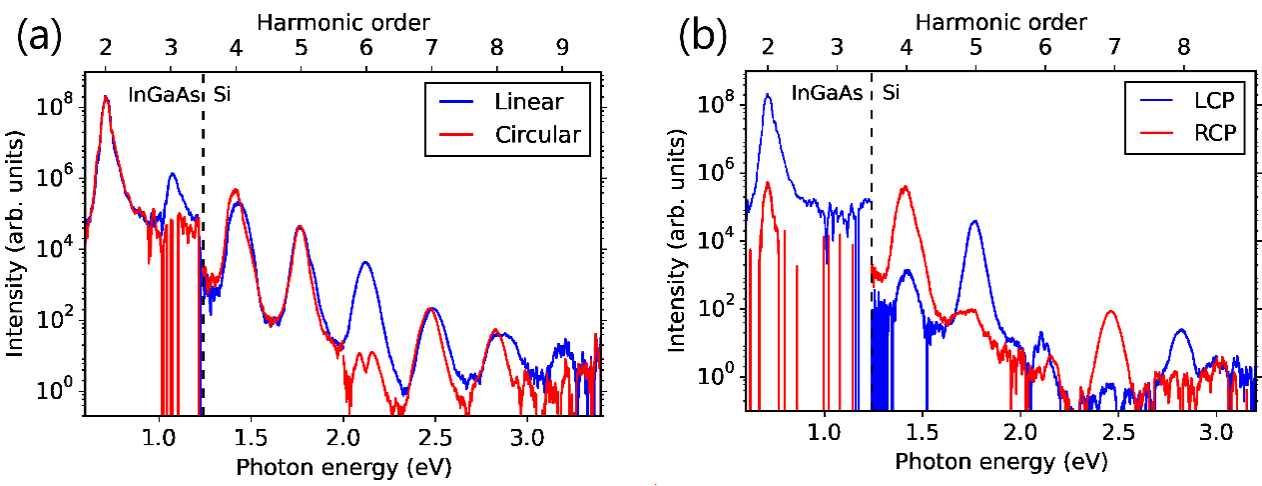

Fig. 2. (a) HH spectra with linear (blue) and circular (red) MIR drive pulses. (b) LCP (blue) and RCP (red) components of the HH spectrum with RCP drive pulses.

\section{References}

1. A. Fleischer, O. Kfir, T. Diskin, P. Sidorenko, and O. Cohen, Nat. Photonics 8, 543 (2014).

2. D. D. Hickstein et al., Nat. Photonics 9, 743 (2015).

3. C. L. Tang and H. Rabin, Phys. Rev. B 3, 4025 (1971).

4. O. E. Alon, V. Averbukh, and N. Moiseyev, Phys. Rev. Lett. 80, 3743 (1998).

5. W. K. Burns and N. Bloembergen, Phys. Rev. B 4, 3437 (1968).

6. K. Konishi et al., Phys. Rev. Lett. 112, 135502 (2014).

7. Y S. You, D. A. Reis, and S. Ghimire, Nat. Phys. 13, 345 (2017).

8. N. Saito et al., Optica 4, 1333 (2017). 